\title{
CORRECTION
}

\section{Correction to: The calculation models of vapor-liquid equilibrium, enthalpy and entropy for R152a/R1234ze(E)}

\author{
Linghao Feng ${ }^{1} \cdot$ Biao $\mathrm{Li}^{1} \cdot$ Yuande Dai $^{1}$ (1)
}

Published online: 16 February 2021

(c) Iranian Chemical Society 2021

\section{Correction to: Journal of the Iranian Chemical Society https://doi.org/10.1007/s13738-021-02155-6}

Through this correction article, title typo error is corrected.

The original article was corrected.

The original article can be found online at https://doi.org/10.1007/ s13738-021-02155-6.

Yuande Dai

dydncutg@qq.com

1 School of Mechanical and Electrical Engineering, Nanchang University, Nanchang 330031, Jiangxi Province, China 